\title{
Supplement
}

\section{Prevalence of disorders in preweaned dairy calves from 731 dairies in Germany:}

A cross-sectional study

DOI: https://doi.org/10.3168/jds.2021-20283

Supplement 1. Overall Prevalence of symptoms for 14,164 pre-weaned dairy calves.

\begin{tabular}{lcccc}
\hline \multirow{2}{*}{ Symptom } & \multicolumn{2}{c}{ Crude $^{1}$} & Region-adjusted & Survey-adjusted \\
& \multicolumn{1}{c}{$\mathrm{n}^{4}$} & $\%(95 \% \mathrm{CI})$ & $\%(95 \% \mathrm{CI})$ & $\%(95 \% \mathrm{CI})$ \\
\hline Fever (F) & $1006^{\mathrm{a}}$ & $7.1(6.7-7.6)$ & $7.2(6.3-8.1)$ & $7.2(6.6-7.9)$ \\
Under temperature (UT) & $447^{\mathrm{a}}$ & $3.2(2.8-3.5)$ & $2.2(0.0-4.5)$ & $1.5(1.2-1.8)$ \\
Aggravated breathing (AB) & $5281^{\mathrm{b}}$ & $37.4(36.6-38.3)$ & $44.5(28.4-60.6)$ & $49.7(48.4-50.9)$ \\
Attenuated breathing (ATB) & $41^{\mathrm{b}}$ & $0.3(0.2-0.4)$ & $0.2(0.0-0.5)$ & $0.2(0.0-0.3)$ \\
Abnormal sounds (AS) & $548^{\mathrm{b}}$ & $3.9(3.5-4.3)$ & $4.5(2.5-6.5)$ & $5.0(4.4-5.6)$ \\
Tubular breathing (TB) & $315^{\mathrm{b}}$ & $2.2(2.0-2.5)$ & $2.1(1.5-2.7)$ & $2.0(1.6-2.4)$ \\
Liquid or soft faeces (LSF) & $2680^{\mathrm{c}}$ & $19.5(18.8-20.2)$ & $18.5(13.8-23.2)$ & $17.8(16.8-18.8)$ \\
Navel abnormalities (NA) & $2985^{\mathrm{d}}$ & $21.1(20.4-21.9)$ & $20.8(8.1-33.5)$ & $20.4(19.5-21.3)$ \\
Unequal loading of limbs (UL) & $119^{\mathrm{e}}$ & $0.8(0.7-1.1)$ & $1.0(0.5-1.6)$ & $1.2(0.9-1.5)$ \\
\hline
\end{tabular}

${ }^{1}$ Actually measured data.

${ }^{2}$ Region-adjusted prevalence (linear mixed model; weighting was equal in all regions).

${ }^{3}$ Survey adjusted prevalence (bootstrap method; weighting was according to the number of calves in the region).

${ }^{4}$ Calves with clinical signs.

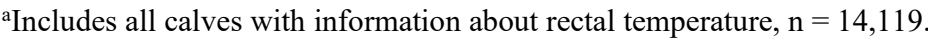

${ }^{b}$ Includes all calves with information about auscultation of the lungs, $n=14,117$.

'Includes all calves with information about faeces, $\mathrm{n}=13,731$.

${ }^{\mathrm{d} I n c l u d e s}$ all calves with information about navel palpation, $\mathrm{n}=14,115$.

e'Includes all calves with information about weight bearing, $\mathrm{n}=14,094$. 
Supplement 2. Overall Prevalence of disorders and multimorbidity for 13,659 pre-weaned dairy calves.

\begin{tabular}{|c|c|c|c|c|}
\hline \multirow[b]{2}{*}{ Disorder } & \multicolumn{2}{|r|}{ Crude $^{1}$} & \multirow{2}{*}{$\begin{array}{c}\text { Region-adjusted }^{2} \\
\%(95 \% \mathrm{CI})\end{array}$} & \multirow{2}{*}{$\begin{array}{c}\text { Survey-adjusted }^{3} \\
\%(95 \% \mathrm{CI})\end{array}$} \\
\hline & $\mathrm{n}^{4}$ & $\%(95 \% \mathrm{CI})$ & & \\
\hline Respiratory Disease (RD) & 1,100 & $8.1(7.6-8.6)$ & $8.7(6.7-10.7)$ & $9.4(8.6-10.2)$ \\
\hline RD_Multimorbidity ${ }^{5}$ & $348^{6}$ & $2.5^{6}(2.2-2.9)$ & $2.7^{6}(2.1-3.3)$ & $3.0^{6}(2.4-3.4)$ \\
\hline Diarrhea (D) & 2,670 & $19.6(18.8-20.3)$ & $18.5(13.7-23.2)$ & $17.9(16.8-18.9)$ \\
\hline D_Multimorbidity ${ }^{5}$ & $787^{6}$ & $5.8^{6}(6.3-6.2)$ & $5.4^{6}(4.2-6.6)$ & $5.0^{6}(4.4-5.6)$ \\
\hline Abnormal Weight & 116 & $0.8(0.7-1.1)$ & $1.0(0.5-1.6)$ & $1.2(0.9-1.6)$ \\
\hline \multicolumn{5}{|l|}{ Bearing (AWB) } \\
\hline AWB_Multimorbidity ${ }^{5}$ & $59^{6}$ & $0.4^{6}(0.3-0.6)$ & $0.4^{6}(0.3-0.6)$ & $0.5^{6}(0.3-0.7)$ \\
\hline Omphalitis (O) & 2,680 & $21.1(20.3-21.8)$ & $20.9(7.8-33.9)$ & $20.3(19.4-21.3)$ \\
\hline O_Multimorbidity ${ }^{5}$ & $819^{6}$ & $6.0^{6}(5.6-6.5)$ & $5.9^{6}(3.6-8.1)$ & $5.6^{6}(5.1-6.3)$ \\
\hline Multimorbidity (M) & 987 & $7.2(6.8-7.7)$ & $7.1(5.7-8.5)$ & $6.9(6.2-7.5)$ \\
\hline
\end{tabular}

${ }^{1}$ Actually measured data.

${ }^{2}$ Region-adjusted prevalence (linear mixed model; weighting was equal in all regions).

${ }^{3}$ Survey adjusted prevalence (bootstrap method; weighting was according to the number of calves in the region).

${ }^{4}$ Number of calves with clinical signs of diseases.

${ }^{5}$ Calves with additional characteristic clinical signs of other disorders.

${ }^{6}$ Each subset of superordinate group: RD, D, AWB and O. 
Supplement 3. Age in days of pre-weaned dairy calves at day of the farm visit depending on the most common breeds and sex stratified by region.

\begin{tabular}{|c|c|c|c|c|}
\hline \multicolumn{5}{|c|}{ 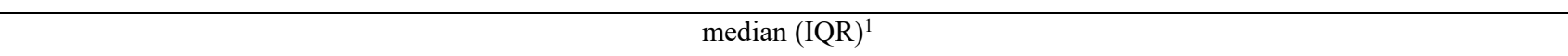 } \\
\hline & & & Region $^{2}$ & \\
\hline & Overall & North & East & South \\
\hline \multicolumn{5}{|l|}{ male } \\
\hline Holstein Friesian & $14.0(8.0,39.0)$ & $23.0(9.0,55.0)$ & $13.0(8.0,31.0)$ & $20.0(11.8,37.25)$ \\
\hline Simmental & $24.0(11.0,38.0)$ & $31.0(13.5,44.5)$ & $16.0(10.0,28.5)$ & $23.5(11.0, \quad 38.0)$ \\
\hline Brown Swiss & $20.0(7.0,35.5)$ & n.a. ${ }^{3}$ & $64.0(64.0,64.0)$ & $20.0(7.0,32.8)$ \\
\hline Red Holstein & $21.0(9.0,56.0)$ & $39.0(10.0,62.0)$ & $12.5(8.0,20.8)$ & n.a. ${ }^{3}$ \\
\hline Crossbreed milk x beef ${ }^{4}$ & $21.0(10.0,48.0)$ & $26.0(10.0,70.0)$ & $15.0(9.0,38.0)$ & $21.0(9.0,25.0)$ \\
\hline Crossbreed milk x milk ${ }^{5}$ & $31.0(13.0,53.0)$ & $26.0(10.0,70.0)$ & $15.0(9.0,38.0)$ & $21.0(9.0,25.0)$ \\
\hline \multicolumn{5}{|l|}{ female } \\
\hline Holstein Friesian & $44.0(23.0,65.0)$ & $48.0(25.0,71.0)$ & $42.0(22.0,64.0)$ & $35.5(20.8, \quad 57.5)$ \\
\hline Simmental & $40.0(20.0,66.0)$ & $36.5(16.3,61.0)$ & $43.5(24.8,67.0)$ & $40.0(20.0, \quad 66.0)$ \\
\hline Brown Swiss & $51.0(24.0,76.5)$ & $46.0(30.0,69.5)$ & $24.0(23.0,39.0)$ & $51.0(24.0, \quad 7.0)$ \\
\hline Red Holstein & $43.0(20.0,71.0)$ & $46.5(19.0,75.3)$ & $40.0(21.0,64.0)$ & $104.0(90.5,117.5)$ \\
\hline Crossbreed milk x beef ${ }^{3}$ & $31.0(12.0,61.0)$ & $29.0(11.0,76.5)$ & $33.0(12.0,57.3)$ & $25.0(14.0,47.8)$ \\
\hline Crossbreed milk x milk ${ }^{4}$ & $38.5(20.3,58.0)$ & $45.0(28.0,58.0)$ & $34.5(19.0,58.0)$ & $47.0(22.0, \quad 88.0)$ \\
\hline
\end{tabular}

${ }^{1}$ interquartile range

${ }^{2}$ Norhern region: medium-sized farms (median 90 milking cows, often full-time family business, partly with external workers). Eastern region: large farms (median 251 milking cows, agricultural enterprise with employees). Southern region: small farms (median 39 milking cows, often traditional family business, some of which are part-time businesses).

${ }^{3}$ Not available.

${ }^{4}$ Cross between a dairy and a beef breed.

${ }^{5}$ Cross between 2 different dairy breeds. 